\title{
Small-scale dynamo action during the formation of the first stars and galaxies
}

\section{The ideal MHD limit}

\author{
D. R. G. Schleicher ${ }^{1,2}$, R. Banerjee ${ }^{3}$, S. Sur ${ }^{3}$, T. G. Arshakian ${ }^{4}$, R. S. Klessen ${ }^{3,5}$, R. Beck ${ }^{4}$, and M. Spaans ${ }^{6}$ \\ 1 ESO, Karl-Schwarzschild-Strasse 2, 85748 Garching bei München, Germany \\ e-mail: dschleic@eso.org \\ 2 Leiden Observatory, Leiden University, PO Box 9513, 2300 RA Leiden, The Netherlands \\ 3 Zentrum für Astronomie der Universität Heidelberg, Institut für Theoretische Astrophysik, Albert-Ueberle-Str. 2, 69120 Heidelberg, \\ Germany \\ 4 Max-Planck-Institut für Radioastronomie, Auf dem Hügel 69, 53121 Bonn, Germany \\ 5 Kavli Institute for Particle Astrophysics and Cosmology, Stanford University, Menlo Park, CA 94025, USA \\ ${ }^{6}$ Kapteyn Astronomical Institute, University of Groningen, PO Box 800, 9700 AV, Groningen, The Netherlands
}

Received 9 June 2010 / Accepted 23 August 2010

\section{ABSTRACT}

\begin{abstract}
We explore the amplification of magnetic seeds during the formation of the first stars and galaxies. During gravitational collapse, turbulence is created from accretion shocks, which may act to amplify weak magnetic fields in the protostellar cloud. Numerical simulations showed that such turbulence is sub-sonic in the first star-forming minihalos, and highly supersonic in the first galaxies with virial temperatures larger than $10^{4} \mathrm{~K}$. We investigate the magnetic field amplification during the collapse both for Kolmogorov and Burgers-type turbulence with a semi-analytic model that incorporates the effects of gravitational compression and small-scale dynamo amplification. We find that the magnetic field may be substantially amplified before the formation of a disk. On scales of $1 / 10$ of the Jeans length, saturation occurs after $\sim 10^{8}$ yr. Although the saturation behaviour of the small-scale dynamo is still somewhat uncertain, we expect a saturation field strength of the order $\sim 10^{-7} n^{0.5} \mathrm{G}$ in the first star-forming halos, with $n$ the number density in cgs units. In the first galaxies with higher turbulent velocities, the magnetic field strength may be increased by an order of magnitude, and saturation may occur after $10^{6}-10^{7} \mathrm{yr}$. In the Kolmogorov case, the magnetic field strength on the integral scale (i.e. the scale with most magnetic power) is higher due to the characteristic power-law indices, but the difference is less than a factor of 2 in the saturated phase. Our results thus indicate that the precise scaling of the turbulent velocity with length scale is of minor importance. They further imply that magnetic fields will be significantly enhanced before the formation of a protostellar disk, where they may change the fragmentation properties of the gas and the accretion rate.
\end{abstract}

Key words. dark ages, reionization, first stars - magnetic fields - dynamo - turbulence - stars: Population III - stars: formation

\section{Introduction}

The formation of the first stars is generally regarded as a welldefined problem, as the initial conditions at $z \sim 100$ can be derived accurately from CMB data (e.g. Komatsu et al. 2009) using linear theory (Bertschinger 1998) and the chemistry is primordial and well-understood (e.g. Abel et al. 1997; Galli \& Palla 1998; Stancil et al. 1998; Omukai 2001; Yoshida et al. 2006; Schleicher et al. 2008b; Glover \& Savin 2009). In addition, it is often assumed that magnetic fields are not yet present and that the hydrodynamical equations are sufficient to describe the star formation process.

This assumption is not neccessarily true. Indeed, a variety of mechanisms exist to create strong magnetic fields during inflation, the electroweak or the QCD phase transition (see e.g. Grasso \& Rubinstein 2001, for a review). By means of the inverse-cascade, the magnetic power of these fields may have been shifted to larger scales in case of non-zero helicity (Brandenburg et al. 1996; Christensson et al. 2001; Banerjee \& Jedamzik 2004). Strong primordial fields would have profound implications concerning the thermodynamics of the postrecombination universe, reionization and the formation of the first stars (Sethi \& Subramanian 2005; Machida et al. 2006; Tashiro \& Sugiyama 2006; Tashiro et al. 2006; Schleicher et al. 2008a, 2009a,b).

In this paper, we will however explore the limiting case in which extremely weak seed fields have been produced before recombination. In such a case, the dominant contribution to the magnetic field strength comes from astrophysical processes after recombination. Cosmological MHD simulations including an approximate treatment of the Biermann battery term suggest that the Biermann battery could create seed fields of the order $10^{-18} \mathrm{G}$ in the IGM at $z=20$ (Xu et al. 2008). Additional seed fields may be created via the Weibel instability in shocks (Schlickeiser \& Shukla 2003; Medvedev et al. 2004; Lazar et al. 2009). The importance of dynamos in cosmic sheets has early been recognized by Pudritz \& Silk (1989). The simulations of $\mathrm{Xu}$ et al. (2008) run from cosmological scales to the protostellar collapse phase in a primordial minihalo. In such a situation, the following mechanisms are available to amplify the magnetic field:

- gravitational compression of the magnetic field,

- the small-scale turbulent dynamo which amplifies seed magnetic fields, 
- large-scale dynamos in protostellar and galactic disks, - the magneto-rotational instability (MRI).

Gravitational compression under spherical symmetry leads to an increase of the magnetic field strength with $n^{2 / 3}$, where $n$ denotes the number density of the gas. If the collapse proceeds preferentially along one axis, for instance because of rotation or strong magnetic fields, the scaling is closer to $n^{0.5}$. In realistic cases, often intermediate values are found (Machida et al. 2006; Banerjee et al. 2008). This amplification mechanism has also been identified in the simulation of Xu et al. (2008).

Large-scale dynamos typically require the presence of a galactic or protostellar disk and act on relatively long timescales (see Brandenburg \& Subramanian 2005, for a review). In such a disk, an exponential growth may also be obtained from the magnetorotational instability (MRI, see Balbus \& Hawley 1991), which may seed other large-scale dynamos with turbulence (Tan \& Blackman 2004; Silk \& Langer 2006). However, the length scale of the fastest growing mode decreases for decreasing field strengths, and in the presence of a viscous cutoff length, such amplification may not be possible. As a result, a minimal field strength is required to drive the MRI in a protostellar disk (see Tan \& Blackman 2004; Silk \& Langer 2006, for a detailed discussion).

A critical condition for any dynamo growth is that the ideal MHD approximation is applicable. Maki \& Susa (2004, 2007) investigated this question using detailed models for magnetic energy dissipation via Ohmic and ambipolar diffusion to show that the magnetic field is frozen into the gas unless it is very strong. An approximate fit to their results yields a critical field strength of about $B \leq 10^{-5}\left(n / 10^{3} \mathrm{~cm}^{-3}\right)^{0.55} \mathrm{G}$. Due to the subtle effects of lithium chemistry, the ionization degree does not drop exponentially at densities of $\sim 10^{9} \mathrm{~cm}^{-3}$, but stays almost constant with increasing density. The more recent study by Glover \& Savin (2009) finds even higher ionization degrees at these densities. This implies that the ideal MHD approximation can be used during the collapse phase to describe the interaction of magnetic fields with matter.

Deviations from this behaviour may however occur on very small scales, where ambipolar and Ohmic diffusion become increasingly important. Estimates based on the non-ideal MHD models of Pinto et al. (2008); Pinto \& Galli (2008) imply that, even if the magnetic field is in equipartition with the gas, ambipolar diffusion is important only on scales 4 orders of magnitudes smaller than the Jeans length, and Ohmic dissipation occurs only on even smaller scales. As Ohmic and ambipolar diffusion depend on the field strength itself, these scales will be significantly smaller for weaker magnetic fields, so that the ideal MHD approximation can be savely applied. This implies that the magnetic Reynolds number $\mathrm{Rm}=v l / \eta$ varies strongly during the growth of the magnetic field, but always fulfills the condition $\mathrm{Rm} \ggg 1$ and $\operatorname{Pr}_{\mathrm{M}}=v / \eta>1$. Detailed calculations concerning the ambipolar and Ohmic diffusion scales will be presented in a companion paper, in which we make use of the ionization degree obtained from a numerical simulation to provide an updated calculation of these scales for different field strengths.

Gravitational collapse is generally accompanied by the presence of turbulence (Klessen \& Hennebelle 2010), which may for instance be described by the theory of Kolmogorov (1941). Numerical simulations show that primordial star formation during the collapse phase occurs in a self-similar fashion, where the density profile at a given time is always well-described by a Bonner-Ebert sphere with a flat central density core (Abel et al. 2002; Bromm \& Loeb 2003; Yoshida et al. 2008). Similar results have been found for present-day star formation (e.g. Banerjee et al. 2006; Banerjee \& Pudritz 2007). The gas falling on these central cores leads to weak shocks up to Mach 1, which drive turbulence in the central density core. This is reflected in the inhomogeneities in the central core and the sub-Keplerian angular momentum profiles, as reported by Abel et al. (2002); Bromm \& Loeb (2003); Yoshida et al. (2008). Under such conditions, a strong tangled magnetic field may be generated already during the collapse phase by the small-scale dynamo that was originally proposed by Kazantsev (1968).

This dynamo provides a very generic means of amplifying magnetic fields and was also proposed to be important in the large-scale structure of the universe (Ryu et al. 2008). The field amplification is due to the random stretching and folding of the magnetic field lines in a turbulent random flow. In the kinematic regime, the field grows typically on the eddy turnover time, $t_{\text {ed }}=l / v$ where $l$ is a typical turbulent length scale and $v$ is the turbulent velocity. In the context of galaxy formation, Beck et al. (1994) proposed that it is the small-scale dynamo that produces the seeds for galactic large-scale dynamos. As pointed out by Arshakian et al. (2009), the small-scale dynamo can effectively amplify weak seed magnetic fields by $\sim 13$ orders of magnitude on a timescale $\sim 300$ million years in the first galaxies. Similar results were obtained by de Souza \& Opher (2010) from a direct solution of Kazantsev's equation.

Capturing such dynamos in numerical simulations of protostellar collapse is extremely challenging, as it requires that the turbulent cascade is well-resolved and well-separated from the scale where MHD turbulence is numerically dissipated. State of the art numerical simulations of turbulence require a spatial resolution of at least $512^{3}$ for a marginally resolved inertial range (Federrath et al. 2008, 2010). Numerical simulations following protostellar collapse, on the other hand, typically resolve the Jeans length and thus the high density region with about 16 cells, rendering them unable to capture the potential amplification via the turbulent dynamo. Federrath et al. (2010) showed that at least 30 grid cells are required to resolve turbulent vortices. Simulations as performed by Xu et al. (2008) therefore cannot resolve the turbulence in the central core.

In this paper, we explore the implications of the small-scale dynamo during the gravitational collapse phase within a semianalytic framework, applied to the formation of the first stars and galaxies. We first review the theoretical background and numerical evidence for the small-scale dynamo in Sect. 2, and present a set of analytic estimates. In Sect. 3, we develop a quantitative model concerning the small-scale dynamo action during the collapse process. This model is applied in Sect. 4 both to minihalos and atomic cooling halos, taking into account the amount of turbulence that was found in numerical simulations. Phenomenological consequences from the generation of such magnetic fields are discussed in Sect. 5. In a companion paper (Sur et al. 2010), we present numerical simulations confirming the importance of dynamo amplification during gravitational collapse.

\section{The small-scale dynamo}

In this section, we introduce the small-scale dynamo by sketching its analytic derivation as well as numerical simulations that examined its efficiency, to make these results accessible to a broader community. This will be combined with a summary on the most important results and first estimates concerning its importance in the first galaxies in Sect. 2.3. Readers that are already 
D. R. G. Schleicher et al.: Small-scale dynamo action during the formation of the first stars and galaxies.I.

familiar with dynamo theory or only interested in the main results may thus directly proceed from Sect. 2.3.

\subsection{Analytical arguments}

The small-scale dynamo was introduced by Kazantsev (1968) in an analytic framework, which was improved subsequently in various works (e.g. Subramanian 1998, 1999; Brandenburg \& Subramanian 2005; Arshakian et al. 2009). For a detailed review we particularly recommend Brandenburg \& Subramanian (2005), as we can sketch here only the main steps in deriving the main properties of this dynamo. The induction equation of the magnetic field $\boldsymbol{B}$ is given as

$$
\frac{\partial \boldsymbol{B}}{\partial t}=\nabla \times(\boldsymbol{v} \times \boldsymbol{B}-\eta \nabla \times \boldsymbol{B})
$$

where $v$ is the velocity field of the fluid and $\eta$ the Ohmic or ambipolar resistivity. The velocity field is decomposed into a stochastic field $\boldsymbol{v}_{\mathrm{T}}$ and a drift component $\boldsymbol{v}_{\mathrm{D}}$. The latter describes the change of the fluid velocity due to the Lorentz force. Such a decomposition is reasonable as long as the kinetic energy dominates over the magnetic energy. As we will see, this is always the case in the regime where the small-scale dynamo is operational. In principle, the drift velocity depends on the complex history of field lines that a certain fluid element has seen during its history. To make the problem analytically tractable, it is generally assumed that most of the history averages out due to the tangledness of the magnetic field. Then, the drift velocity points at least approximately into the direction of the instantaneous Lorentz force. It is generally approximated as (Kazantsev 1968; Subramanian 1998; Brandenburg \& Subramanian 2005)

$\boldsymbol{v}_{\mathrm{D}}=\frac{\tau}{4 \pi \rho}[(\nabla \times \boldsymbol{B}) \times \boldsymbol{B}]$,

where $\tau$ is the typical response time of the magnetic field and $\rho$ the gas density. The stochastic field component $\boldsymbol{v}_{\mathrm{T}}$, on the other hand, is assumed to be an isotropic, homogeneous, Gaussian random velocity field with zero mean. We further adopt the Markovian approximation, assuming that its correlation function is given as (Kazantsev 1968; Subramanian 1998; Brandenburg \& Subramanian 2005)

$$
\left\langle v_{\mathrm{T}}^{i}(\boldsymbol{x}, t) v_{\mathrm{T}}^{j}(\boldsymbol{y}, s)\right\rangle=T^{i j}(r) \delta(t-s),
$$

with $r=|\boldsymbol{x}-\boldsymbol{y}|$ and $\delta$ denoting the delta distribution function. The matrix $T^{i j}$ can be decomposed in a longitudinal part $T_{\mathrm{L}}(r)$, a transverse part $T_{\mathrm{N}}(r)$ and a helical part $C(r)$. In the presence of homogeneous, isotropic and Gaussian turbulence, a weak seed magnetic field will be dragged with the fluid and obey the same properties. We denote the equal-time two-point correlation function of the magnetic field as $M^{i j}(r, t)=\left\langle B^{i}(\boldsymbol{x}, t) B^{j}(\boldsymbol{y}, t)\right.$ and decompose it into a longitudinal component $M_{\mathrm{L}}(r, t)$, a transverse component $M_{\mathrm{N}}(r, t)$ and a helical component $H(r, t)$. Defining $r^{i}=x^{i}-y^{i}$ and introducing the Kronecker delta $\delta^{i j}$ and the totally antisymmetric tensor $\epsilon_{i j k}$, one can write (Subramanian 1998; Brandenburg \& Subramanian 2005)

$M^{i j}=M_{\mathrm{N}}\left[\delta^{i j}-\frac{r^{i} r^{j}}{r^{2}}\right]+M_{\mathrm{L}} \frac{r^{i} r^{j}}{r^{2}}+H \epsilon_{i j k} r^{k}$.

As the magnetic field is divergence free, one can show that

$M_{\mathrm{N}}=\frac{1}{2 r} \frac{\partial r^{2} M_{\mathrm{L}}}{\partial r}$.
From the induction Eq. (1), one can derive the following evolution equation for $M_{\mathrm{L}}$ and $H$ (Kazantsev 1968; Subramanian 1998; Brandenburg \& Subramanian 2005):

$$
\begin{aligned}
\frac{\partial M_{\mathrm{L}}}{\partial t} & =\frac{2}{r^{4}} \frac{\partial}{\partial r}\left(r^{4} \kappa_{\mathrm{N}} \frac{\partial M_{\mathrm{L}}}{\partial r}\right)+G M_{\mathrm{L}}-4 \alpha_{\mathrm{N}} H \\
\frac{\partial H}{\partial t} & =\frac{1}{r^{4}} \frac{\partial}{\partial r}\left(r^{4} \frac{\partial}{\partial r}\left(2 \kappa_{\mathrm{N}} H+\alpha_{\mathrm{N}} M_{\mathrm{L}}\right)\right) .
\end{aligned}
$$

These equations include a diffusion term with coefficient $\kappa_{\mathrm{N}}=$ $\eta+T_{\mathrm{L}}(0)-T_{\mathrm{L}}(r)+2 a M_{\mathrm{L}}(0, t)$. The coefficient $\alpha_{\mathrm{N}}=2 C(0)-$ $2 C(r)-4 a H(0, t)$ describes the strength of the $\alpha$ effect, and the term involving $G=-4\left(\left(T_{\mathrm{N}} / r\right)^{\prime}+\left(r T_{\mathrm{L}}\right)^{\prime} / r^{2}\right)$ describes the rapid generation of magnetic fluctuations by velocity shear. The prime denotes the derivative with respect to $r$.

We will now focus on the case of nonhelical turbulence, with $C(r)=0$ and $H(r, t)=0$, as it is more straightforward to be treated analytically. The formalism can however be applied to helical turbulence. In that case, the turbulent dynamo acts in the same way on small-scales, but creates additional large-scale correlations that can act as seed fields for a large-scale dynamo (Subramanian 1999). We are looking for eigenmode solutions of Eq. (6) and thus make the ansatz $\Psi(r) \exp (2 \Gamma t)=r^{2} \sqrt{\kappa_{N}} M_{\mathrm{L}}$. Insertion in Eq. (6) leads to a time independent equation which formally resembles the Schrödinger equation of quantum mechanics (Kazantsev 1968; Subramanian 1998; Brandenburg \& Subramanian 2005):

$-\Gamma \Psi=-\kappa_{\mathrm{N}} \frac{d^{2} \Psi}{d r^{2}}+U_{0}(r) \Psi$.

Under the assumption that the velocity field is locally divergence-free, one can show that $U_{0}(r)=T_{\mathrm{L}}^{\prime \prime}+\left(2 T_{\mathrm{L}}^{\prime} / r\right)+\kappa_{\mathrm{N}}^{\prime \prime} / 2-$ $\left(\kappa_{\mathrm{N}}^{\prime}\right)^{2} /\left(4 \kappa_{\mathrm{N}}\right)+2 \kappa_{\mathrm{N}} / r^{2}$. Of course, the assumption of a divergencefree velocity field is only approximately true for compressible gas. However, a turbulent velocity field can always be decomposed into a divergence-free and a divergent component, and the divergent component is just neglected to simplify the analytic treatment. As discussed in the next subsection, numerical simulations that generally include both components still find the same results concerning the magnetic field amplification timescale.

As a boundary condition, we adopt $\Psi \rightarrow 0$ for $r \rightarrow 0, \infty$. The solution of Eq. (8) depends on the stability of flow of the magnetized fluid, which is depends on the magnetic Reynolds number $\mathrm{Rm}=v l / \eta$, which describes the relative importance of the interaction of the magnetic field with the velocity field compared to Ohmic or ambipolar dissipation. As discussed in the introduction, high magnetic Reynolds numbers can be expected during primordial collapse. For the solutions of Eq. (8), a critical magnetic Reynolds number $\mathrm{Rm}_{\mathrm{cr}} \sim 60$ exists for which the solution corresponds to a bound state with $\Gamma=0$ (Kazantsev 1968; Subramanian 1998, 1999). For $\mathrm{Rm}>\mathrm{Rm}_{\mathrm{cr}}, \Gamma>0$ modes can be excited, leading to an exponential growth of the magnetic field on the eddy turnover timescale $l / v$. Such a magnetic field is then curved on the turbulent length scale $l$. The thickness of the flux ropes is more uncertain and may depend on the critical Reynolds number for the dynamo (Subramanian 1998) or the resistive scales (Schekochihin et al. 2004).

To obtain the value at which the magnetic field saturates, the evolution Eq. (6) must be solved for a stationary state. The analysis shows that the magnetic energy then corresponds to a fraction $\mathrm{Rm}_{\mathrm{cr}}^{-1}$ of the kinetic energy. The maximum magnetic 
field strength that can be obtained in this way is thus given as (Subramanian 1998)

$$
B_{\max }=\sqrt{4 \pi \rho v^{2}} \mathrm{Rm}_{\mathrm{cr}}^{-1 / 2} \text {. }
$$

We note that the saturation field strength is still somewhat uncertain; our main intention here is to show that saturation can be reached. We checked that this conclusion does not depend on the precise fraction for the saturation level adopted here.

\subsection{Confirmation from numerical simulations}

As pointed out above, the complexity of modeling magnetic fields, gas dynamics and their mutual interplay forces one to make simplifying assumptions in an analytic treatment. Thus, numerical simulations are required to test the analytic results. Evidence of small-scale dynamo action has so far been confirmed in numerical simulations of forced MHD turbulence (Haugen et al. 2004b; Schekochihin et al. 2004) both for high as well as low magnetic Prandtl numbers. Such simulations were able to follow the evolution of the magnetic field even in the nonlinear regime when the Lorentz forces become strong enough to saturate the dynamo. In the simulations of Haugen et al. (2004b), exponential growth of the magnetic field commences once the magnetic Reynolds number exceeds a critical value which for $\operatorname{Pr}_{m}=1$ is $R m \sim 35$. This critical value is somewhat larger by a factor 3 compared to the $\mathrm{Rm}_{\mathrm{cr}}$ obtained from the Kazantsev model, which assumes a delta-correlated velocity field. Simulations starting with an initially coherent field find that the magnetic power spectrum follows a $k^{3 / 2}$ slope before saturation sets in. The growth rate of the field increases with $\left(\mathrm{Rm} / \mathrm{Rm}_{\mathrm{cr}}\right)^{1 / 2}$ for higher magnetic Reynolds number. Apart from this, the salient feature of the small-scale dynamo is the presence of highly intermittent and structured fields. This property has been discussed in detail by Schekochihin et al. (2004); Brandenburg \& Subramanian (2005). The magnetic fields appear to be in folds whose length is comparable to the box size and which reverse directions at the resistive scale. The growing magnetic field appears highly intermittent in the sense that it has strong positive(negative) values only in a few places in the simulation domain. These results were confirmed at higher resolution (Haugen et al. 2004a) and for different Mach numbers (Haugen et al. 2004c), making a robust case for the efficiency of the small-scale dynamo.

\subsection{Order-of-magnitude estimates}

In summary, previous investigations found that the small-scale dynamo

- amplifies a magnetic fields on the eddy timescale;

- leads to magnetic fields curved on scales up to the turbulent length scale;

- and a thickness of the flux ropes either given from the resistive scales or the critical Reynolds number for dynamo amplification.

As the analytic procedures discussed in Sect. 2.1 are based on the simplifying assumption that the gas is incompressible, they cannot be directly applied to a situation in which the gas is collapsing. Rather than this, we will focus now on the main results that were obtained with analytical and numerical simulations and develop a new framework in which we can apply them during the star formation process.
In order to assess the potential importance of the small-scale dynamo in the first galaxies, we start with an order-of-magnitude estimate concerning the rapid build-up of magnetic fields. The amount of turbulence in the first galaxies has been studied with numerical simulations by Greif et al. (2008) and Wise \& Abel (2007); Wise et al. (2008). On spatial scales of $200 \mathrm{pc}$, they find typical turbulent velocities of $\sim 20 \mathrm{~km} \mathrm{~s}^{-1}$. To assess how much such turbulence can amplify magnetic fields in the first galaxies, we need to calculate the number of e-foldings within a free-fall time that are available for magnetic field amplification. The free-fall timescale is given as $t_{\mathrm{ff}}=1 / \sqrt{\rho G}$, with $\rho$ the total mass density and $G$ Newton's constant. We assume that the turbulent velocity scales as $v(l) \propto l^{\beta}$, with $\beta=1 / 3$ for Kolmogorov turbulence and $\beta=1 / 2$ for Burgers turbulence, on scales smaller than the injection scale. Kolmogorov turbulence describes a situation where the gas is incompressible and is thus applicable in particular for sub-sonic turbulence, whereas Burgers turbulence describes turbulence in the presence of supersonic shocks, where the gas is quite strongly compressed.

We note that Burgers turbulence should be considered as a highly idealized situation, because numerical simulations show that turbulence even in the supersonic regime always consists of rotational and compressional components of comparable strength (Haugen et al. 2004c; Federrath et al. 2008, 2010), and dedicated studies by Kritsuk et al. (2007), Schmidt et al. (2009) and Federrath et al. (2010) typically find power-laws in between the Burgers and Kolmogorov case. As a side note, we mention that purely irrotational turbulence may not be able to drive the small-scale dynamo at all (Mee \& Brandenburg 2006), whereas realistic turbulence should always have rotational and irrotational components as discussed above.

The eddy timescale is given as $t_{\mathrm{ed}}=l / v$. Considering that the small-scale dynamo should not be amplified above the saturation value given in Eq. (9), the magnetic field strength after a free-fall time is given as

$$
B=\min \left(B_{0} \exp \left(\frac{v}{l \sqrt{\rho G}}\right), B_{\max }\right),
$$

with $B_{0}$ denoting the strenth of the seed field. To estimate the potential impact of the small-scale dynamo, we evaluate Eq. (10) for a baryonic number density of $0.02 \mathrm{~cm}^{-3}$, corresponding to the mean density at virialization. We take into account the contribution of dark matter to the total mass density. According to Greif et al. (2008) and Wise \& Abel (2007); Wise et al. (2008), we assume that the turbulence is injected on scales of $\sim 200 \mathrm{pc}$ with a velocity of $\sim 20 \mathrm{~km} \mathrm{~s}^{-1}$. On smaller scales, we expect that the turbulent velocity follows some typical scaling law as in Kolmogorov or Burgers turbulence. We explore these cases for definiteness. We adopt a seed field of $B_{0} \sim 10^{-20} \mathrm{G}$, somewhat below the value expected from a Biermann battery mechanism (Xu et al. 2008). The results are shown in Fig. 1. In case of Kolmogorov turbulence, the magnetic field is negligible on scales of a few hundred parsec, but increases rapidly towards smaller scales. A maximum is reached on scales of $\sim 80 \mathrm{pc}$, where the field strength has increased by about 13 orders of magnitude. The maximum occurs because the magnetic field has reached the saturation level on this scale. On all smaller scales, the eddy timescale is smaller, decreasing as $l / v \propto l^{2 / 3}$ in the Kolmogorov case. The magnetic field is thus saturated at smaller scales. As the turbulent velocity decreases with decreasing scale, also the saturation value for the magnetic field strength decreases correspondingly, thus explaining the maximum at $\sim 80 \mathrm{pc}$.

For Burgers-type turbulence, the situation is similar. As the turbulent velocity decreases more rapidly with scale, the 
D. R. G. Schleicher et al.: Small-scale dynamo action during the formation of the first stars and galaxies.I.

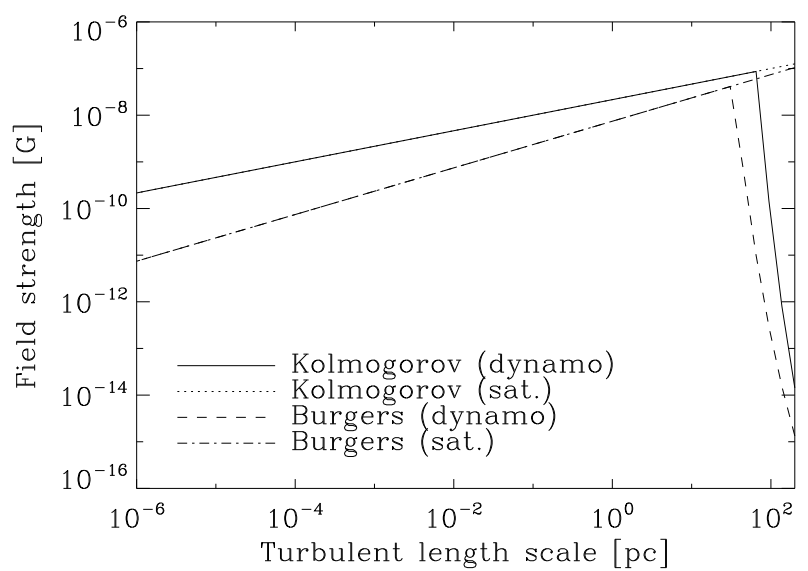

Fig. 1. Estimates on the field strength that can be reached within a freefall time as a function of scale. Shown are both the actual field strength to which the magnetic field is amplified by the small-scale dynamo, as well as the maximum field strength at which dynamo action saturates. This saturation field strength increases continuously as a powerlaw until the scale where turbulence is injected, whereas the actual field strength has a maximum between 10 and $100 \mathrm{pc}$. We consider both Kolmogorov and Burgers turbulence.

magnetic field reaches the saturation level only on somewhat smaller scales of $\sim 30 \mathrm{pc}$. Towards even smaller scales, it decreases more rapidly, as the turbulent velocity field and thus the saturation field strength decreases more rapidly with decreasing length scale.

Of course, this estimate is only approximate, as the magnetic field should also be subject to gravitational compression, which reduces the coherence length but amplifies the field strength. In addition, the density will increase during the gravitational collapse, thus increasing the field strength at which the magnetic field saturates. We also expect that the scale on which the turbulence is injected changes during the collapse. If the magnetic field saturates, additional effects like turbulent decay may come into play. To account for these additional physics, we propose a more detailed model in Sect. 3.

\section{Magnetic fields during turbulent collapse}

In this section we derive a model for the evolution of a turbulent gas cloud with initially weak magnetic fields. For this purpose, a model of turbulence and the evolution of the turbulent spectrum during the gravitational collapse is required. In this section, we first develop such a model on the basis of analytical arguments and numerical results (Wise \& Abel 2007; Greif et al. 2008; Wise et al. 2008). Based on this model, the growth of the magnetic field in the dynamo phase can be calculated. We also discuss the subsequent evolution in the saturated phase, in which the competition between turbulent decay, gravitational compression and dynamo amplification governs the evolution of the magnetic field.

\subsection{Turbulent collapse}

During the galaxy formation process, turbulence is generated by the release of gravitational energy and the infall of accreted gas on the inner, self-gravitating core. Under such conditions, the injection scale of turbulence is usually comparable to the size of the system under consideration (Klessen \& Hennebelle 2010). Here, we focus on the central density core found in numerical simulations of primordial star formation (Abel et al. 2002; Bromm \& Loeb 2003; Yoshida et al. 2008). Its extent is comparable to the Jeans length, which can be derived from the critical mass required to make the gas cloud gravitationally unstable:

$M_{\mathrm{J}}=2 M_{\odot}\left(\frac{c_{\mathrm{s}}}{0.2 \mathrm{~km} \mathrm{~s}^{-1}}\right)^{3}\left(\frac{n}{10^{3} \mathrm{~cm}^{-3}}\right)^{-1 / 2}$.

Here, $c_{\mathrm{s}}$ is the sound speed. This central core is no longer dominated by radial motions, but rather supported by turbulence generated in accretion shocks. Due to the continuous infall of gas, the turbulence will not decay, but is constantly replenished. Radial profiles of the turbulent velocity in the first galaxies indicate that there are some random fluctuations, but the orderof-magnitude of the turbulent velocity does not change during the collapse (Wise \& Abel 2007; Greif et al. 2008; Wise et al. 2008). For turbulence driven by accretion, one expects that the turbulent velocity is comparable to the infall velocity, and for a roughly isothermal density profile, the free-fall velocity is independent of radius. We will therefore assume that, while the injection scale changes during the collapse, the injected velocity stays the same. On scales smaller than the size of the cloud, we expect that the turbulent velocity scales as a $v \propto l^{\beta}$. As above, we will explore both Kolmogorov and Burgers-type turbulence. The evolution of the mean density with time is prescribed as in the one-zone models of Glover \& Savin (2009) and Schleicher et al. (2009c), which follow the evolution of primordial chemistry during the collapse phase.

\subsection{Evolution in the dynamo phase}

As discussed in the introduction, there are two mechanisms that can amplify the magnetic field in the collapse phase: The smallscale dynamo, and gravitational compression. In this subsection, we propose a Lagrangian framework that allows to treat these effects simultaneously. We consider an array of fluctuation length scales $l_{i}$ with a corresponding array of field strength $B_{i}$, and follow both the evolution of the magnetic field strength $B_{i}$ and its corresponding length scale $l_{i}$ over time. For the initial field strength, we adopt a conservative value of $10^{-20} \mathrm{G}$ on all scales.

The initial length scales are chosen with a constant logarithmic spacing, with the largest length scale corresponding to the Jeans length $\lambda_{\mathrm{J}}$, and the smallest length scale corresponding to $10^{-4} \lambda_{\mathrm{J}}$. This range was adopted to make sure that the integral scale always lies well-within the range of scales followed in the code. We have estimated the resistive scale following the framework of Pinto et al. (2008); Pinto \& Galli (2008), finding that it lies well below this value. We follow the evolution of 1000 length scales with their corresponding magnetic field strength. For each length scale $l_{i}$, we calculate the turbulent velocity $v_{i}(t)$ as described in the previous subsection. As the smallscale dynamo amplifies each of these fields on the eddy-turnover timescale, we describe this process by solving the following set of ordinary differential equations (ODEs):

$\frac{\mathrm{d} B_{i}(t)}{\mathrm{d} t}=\frac{B_{i}(t)}{l_{i} / v_{i}}$

In addition, gravitational compression amplifies the magnetic field strength and contracts the corresponding length scale. Under spherically symmetric conditions, the magnetic field strength scales as $B \propto \rho^{2 / 3}$, whereas a more modest increase would be appropriate if the magnetic field could significantly distort the geometry and lead to collapse along one preferred direction. In our model, we consider an initial seed field which 
is too weak to be dynamically important. Further, even after sufficient amplification, the small-scale dynamo will produce a highly tangled magnetic field, which will not change the geometry. We therefore increase the magnetic field strength according to this scaling law after each timestep. In addition, the corresponding length scale will be compressed. For collapse under spherically symmetric conditions, we expect it to scale as $l \propto \rho^{1 / 3}$. During the turbulent collapse, the initial seed field will thus be continuously amplified, while its length scale is compressed by gravity.

As the magnetic field within the collapsing cloud is always distorted by the gravitational collapse, the largest coherence length that can be achieved is always smaller than the Jeans length by some factor $f_{\mathrm{d}}$. The precise value of $f_{\mathrm{d}}$ is uncertain, as this problem has not been examined with numerical simulations yet. Throughout this paper, we adopt a fiducial value of $f_{\mathrm{d}}=0.1$. A variation of this value by a factor of a few will however not change our conclusions significantly. Indeed, even somewhat larger coherence lengths may be possible, but this needs to be explored with numerical simulations. When we determine the integral scale in the subsequent applications, i.e. the scale on which the magnetic field strength is largest, we will thus explicitly ensure that it cannot be larger than $f_{\mathrm{d}} \lambda_{\mathrm{J}}$. When the saturation field strength is reached, we do no longer solve Eq. (12), but switch to the treatment described in the next subsection.

\subsection{Evolution in the saturated phase}

Once the magnetic field $B_{i}$ on scale $l_{i}$ is larger than the saturation value given in Eq. (9), it is no longer amplified by the small-scale dynamo. It is however still amplified by gravitational compression according to $\rho^{2 / 3}$, whereas the saturation field strength only increases as $\rho^{1 / 2}$. It can thus in principle increase above the saturation level. In this case, it is however subject to turbulent decay.

In MHD simulations of decaying turbulence without selfgravity, one typically finds a power-law decay of the total magnetic energy (Smith \& Mac Low 1998; Biskamp \& Müller 1999). For our model, we need a prescription for the magnetic field on a given scale rather than the total magnetic energy. As shown e.g. by Subramanian et al. (2006), the power-law behaviour of the total energy results from an exponential decay of the magnetic field on a given scale, which happens on the eddy-timescale. This is because the integral scale increases during the decay, as the energy on smaller scales dissipates more quickly. Due to the growth of the integral scale, the total energy thus decreases as a power-law.

In the presence of gravity, these effects have not been investigated explicitly. However, it seems likely that the picture of purely decaying turbulence will not hold under these circumstances. In particular, it is likely that the integral scale cannot become larger than a fraction of the Jeans length, which we parametrized above as $f_{\mathrm{d}}$. Once saturation is reached, the integral scale within the central core thus continuously decreases over time.

We therefore do not expect a power-law behaviour as in the case of decaying turbulence without gravity. For the kinetic turbulence, in fact we expect that typical turbulent velocities do not change significantly, although their scale will continuously decrease during the collapse. This is because the turbulence can be continuously replenished by accretion shocks. A magnetic field stronger than the saturation value can however decay. On a given scale, this should occur on the eddy-timescale, similar as for dynamo amplification. We thus describe it as

$$
\frac{\mathrm{d} B_{i}(t)}{\mathrm{d} t}=-\frac{B_{i}(t)}{l_{i} / v_{i}}
$$

For a magnetic field strength above the saturation value, its evolution is thus governed by Eq. (13), while it is governed by Eq. (12) for weaker fields. As a consequence, the magnetic field thus tends to stay close to the saturation value, which increases as $\rho^{1 / 2}$.

\section{Application to the first stars and galaxies}

The model developed above is now well-suited to study the implications of the small-scale dynamo in the first star-forming systems in the early universe. In this section, we apply it to minihalos that may harbor the first stars, and atomic cooling halos which are often considered to be the first galaxies in the universe.

\subsection{Evolution in primordial minihalos}

The first stars are suggested to form in primordial minihalos with $\sim 10^{5}-10^{6} M_{\odot}$ between $z \sim 30$ and $z \sim 15$ (Abel et al. 2002; Bromm \& Larson 2004; Glover 2005; Yoshida et al. 2008). These halos have initial temperatures of a few $1000 \mathrm{~K}$, and an initial ionisation degree of $\sim 2 \times 10^{-4}$, corresponding to the relic electron fraction left after recombination (Seager et al. 1999, 2000). The main cooling mechanism is thus line emission from molecular hydrogen, which forms primarily due to the $\mathrm{H}^{-}$ channel.

The turbulent properties in the first star-forming minihalos have not yet been studied to a satisfactory degree and thus provide a relevant uncertainty in this analysis. The presence of turbulence is however evident from the inhomogeneities in the central cloud cores found in a number of simulations, and the sub-keplerian angular momentum profiles (Abel et al. 2002; Bromm \& Loeb 2003; Yoshida et al. 2008). Tan \& Blackman (2004) mentions the presence of shock-velocities comparable to the sound speed.

In our turbulent collapse model, we will thus assume that a turbulent velocity equal to the sound speed is injected on the Jeans scale, leading to sub-sonic turbulence on the integrale scale of the magnetic field. We consider both Kolmogorov and Burgers-type turbulence.

With the model obtained in Sect. 3, we follow the evolution of the magnetic field spectrum, and plot the evolution of the magnetic field strength on the integral scale, as well as the integral scale as a function of density, in Figs. 2 and 4. In the initial dynamo phase, the magnetic field strength increases rapidly by several orders of magnitude, as the eddy-turnover time on subpc scales is much smaller than the free-fall timescale. We show the evolution during this dynamo-phase in more detail in Fig. 3. The spectrum of the magnetic field during this early phase, at a density of $10 \mathrm{~cm}^{-3}$, is shown in Fig. 5. As one can see, the integral scale is initially small, but increases up to $\sim 3 \mathrm{pc}$ at a density of $\sim 10^{2} \mathrm{~cm}^{-3}$. At that point, the magnetic field is in the saturated phase, where it increases roughly with $n^{0.5}$, while the integral scale decreases as the Jeans scale due to gravitational compression. We find the same qualitative behaviour for the Kolmogorov- and the Burgers-type turbulence, indicating that the precise scaling of turbulent velocity with length scale is of minor importance. In both cases, we find a rapid build-up phase and a subsequent saturation phase. For Burgers-type turbulence, 
D. R. G. Schleicher et al.: Small-scale dynamo action during the formation of the first stars and galaxies.I.

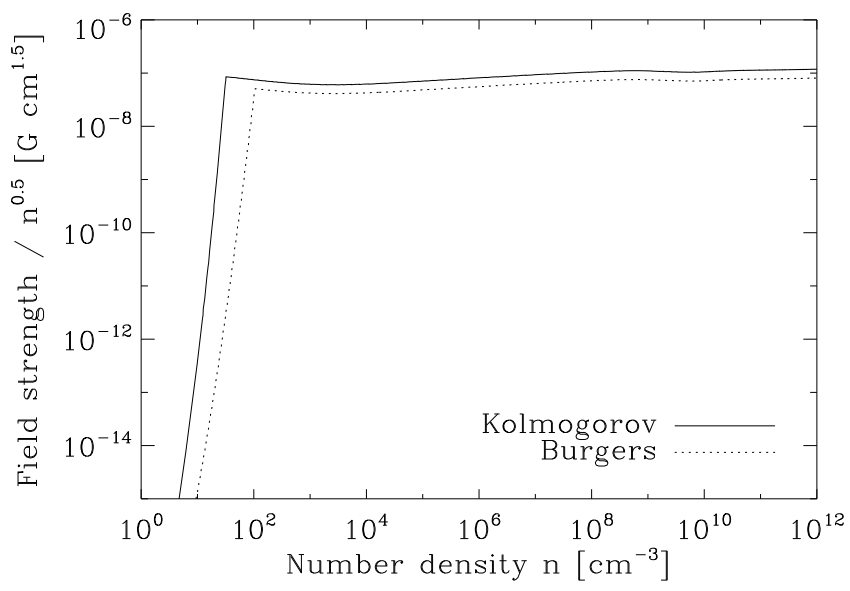

Fig. 2. The evolution of the magnetic field strength on the integral scale during protostellar collapse in a typical minihalo with subsonic turbulent velocities shown as a function of the average density reached in the central core at a given time. We show cases corresponding to Kolmogorov- and Burgers-type turbulence.

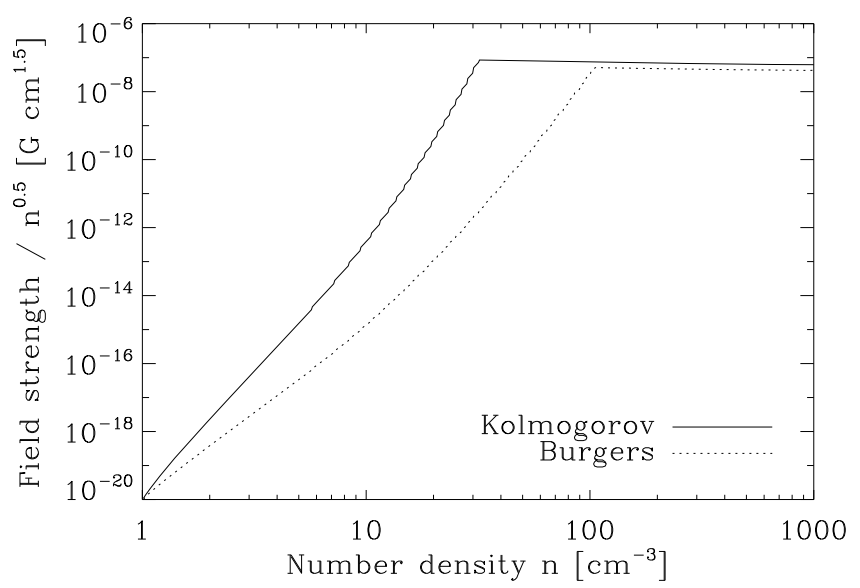

Fig. 3. Magnetic field amplification in the early collapse phase. We show the same quantities as in Fig. 2, with particular focus on the initial phase.

the build-up is just slightly delayed, and the field saturates on a slightly lower level, as the typical velocity on the largest possible integral scale, $f_{\mathrm{d}} \lambda_{\mathrm{J}}$, is somewhat decreased.

In Fig. 6, we show the evolution of magnetic pressure for both cases. We find that the thermal pressure clearly dominates over the magnetic pressure in both cases. Magnetic fields created by the small-scale dynamo may thus not change the protostellar collapse phase significantly. However, they are strong enough to change fragmentation behaviour and binary formation in the disk phase, as discussed in Sect. 5. Magnetic effects therefore need to be considered for a correct assessment of the primordial initial mass function (IMF).

\subsection{Evolution in atomic cooling halos}

Atomic cooling halos are defined as systems with virial temperatures of at least $10^{4} \mathrm{~K}$ and have masses of $M>5 \times 10^{7}[(1+$ $z) / 10]^{-3 / 2} M_{\odot}$. Graviational infall can thus shock-heat the gas to $\sim 10^{4} \mathrm{~K}$, leading to an increased ionisation degree up to $10^{-2}$. Under such conditions, atomic hydrogen becomes an important cooling agent. In addition, the presence of an additional cold accretion mode has been found in numerical simulations

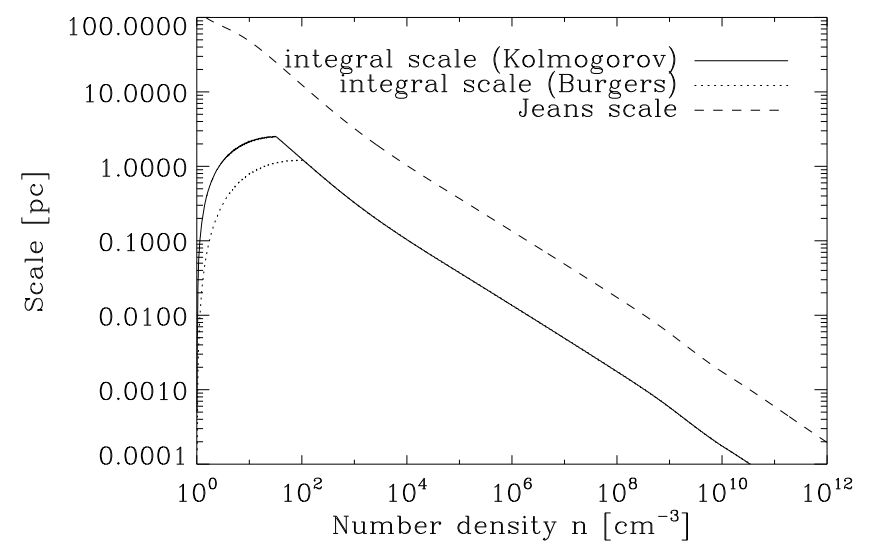

Fig. 4. The evolution of the integral scale of the magnetic field as a function of the average density reached in the central core at a given time during protostellar collapse in a typical minihalo with subsonic turbulent velocities. We show cases corresponding to Kolmogorov- and Burgers-type turbulence and compare with the Jeans scale.

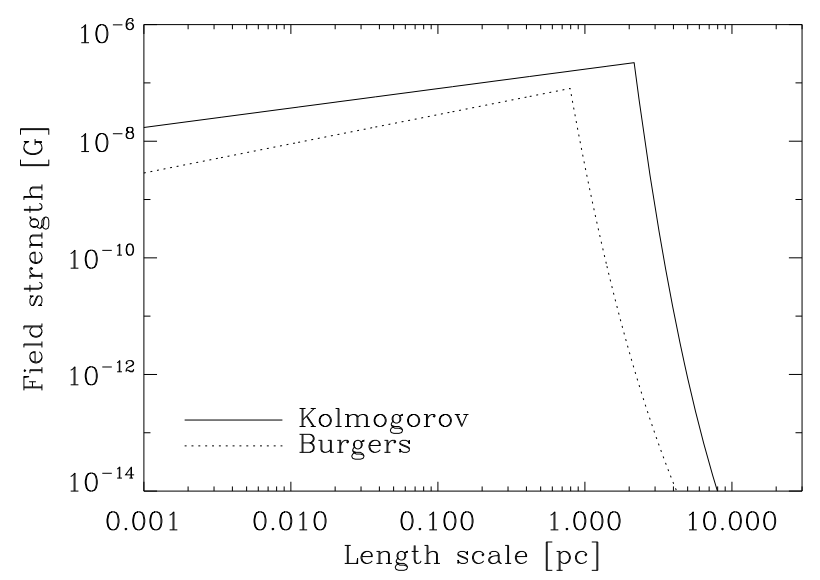

Fig. 5. The magnetic field as a function of scale when an average density of $10 \mathrm{~cm}^{-3}$ is reached in the central core, in a typical minihalo, shown for Kolmogorov- and Burgers-type turbulence.

(Greif et al. 2008), which results from accretion in dense filaments with enhanced fractions of molecular hydrogen. The gas can thus cool efficiently while it is accreted, thus staying at temperatures of a few hundred K. Due to the large column densities, Lyman $\alpha$ photons may be efficiently trapped in these systems (Spaans \& Silk 2006). The presence of a photodissociating background may suppress the $\mathrm{H}_{2}$ abundance and increase the gas temperatures (Omukai 2001). Even the combination of these effects is however not able to prevent the gas temperature from decreasing during the collapse (Schleicher et al. 2010).

As shown by Greif et al. (2008) and Wise \& Abel (2007); Wise et al. (2008), the gas in these systems is subject to supersonic turbulence. They find supersonic turbulence with Mach numbers up to 10 , corresponding to turbulent velocities of $\sim 20 \mathrm{~km} \mathrm{~s}^{-1}$. We will adopt this as a fiducial value for the turbulent velocity on the injection scale and again consider the consequences of Kolmogorov and Burgers-type turbulence.

As shown in Fig. 7, the magnetic field strength increases even more rapidly in this case, due to the supersonic turbulent velocities in atomic cooling halos. The magnetic field saturates at densities still lower than $10 \mathrm{~cm}^{-3}$ and evolves then with $n^{0.5}$, like the saturation scale. As shown in Fig. 8, the integral scale also increases more rapidly, yielding values of $\sim 10 \mathrm{pc}$ at a density of $\sim 3 \mathrm{~cm}^{-3}$. Towards higher densities, its evolution 


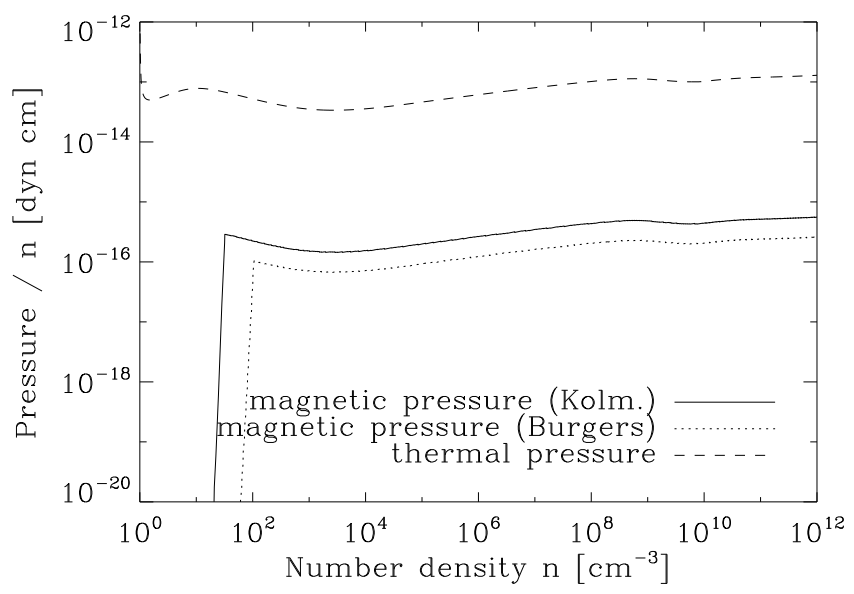

Fig. 6. Thermal and magnetic pressure as a function of the average density reached in the central core at a given time during protostellar collapse in a typical minihalo with subsonic turbulent velocities, for Kolmogorov- and Burgers-type turbulence. In both cases, thermal pressure dominates over the magnetic one.

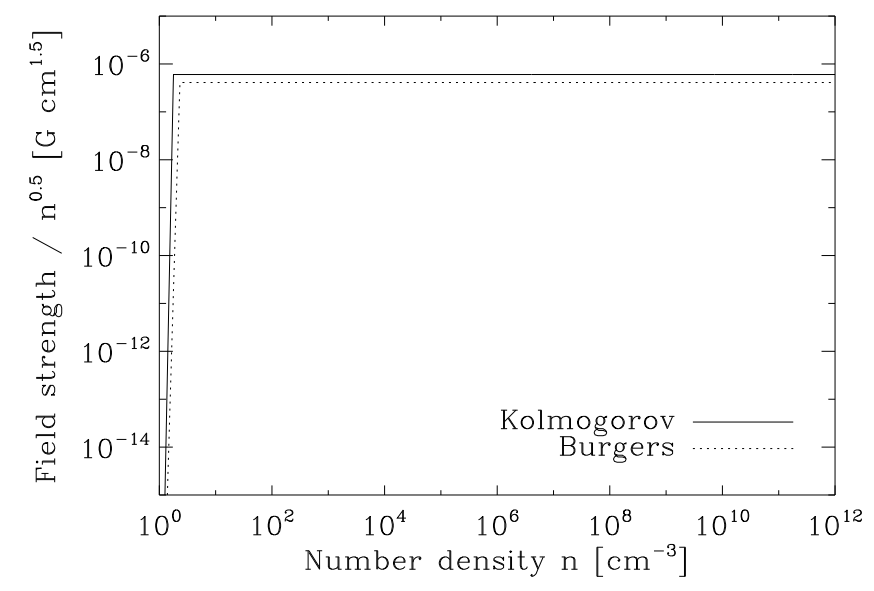

Fig. 7. The evolution of the magnetic field strength on the integral scale during protostellar collapse in an atomic cooling halo with turbulent velocities of $\sim 20 \mathrm{~km} \mathrm{~s}^{-1}$ on the injection scale shown as a function of the average density reached in the central core at a given time. We show cases corresponding to Kolmogorov- and Burgers-type turbulence.

is dictated by gravitational compression. It thus scales as the thermal Jeans length. As for minihalos, the difference between a Kolmogorov and a Burgers-type spectrum is of minor importance, as in both cases the saturation value is rapidly reached.

In Fig. 9, we compare magnetic and thermal pressure in atomic cooling halos. Due to the supersonic turbulent velocities, the saturation field strength is highly increased, and thus also the magnetic pressure. The ratio between the thermal and the magnetic field strength varies between 0.5 and 0.1 . In the presence of additional coolants due to metal-enrichments, this difference will decrease further. As discussed below in more detail, the magnetic field will be highly inhomogeneous in a three-dimensional configuration and may thus be dynamically important locally. The magnetic field may thus change the evolution in the collapse phase and also after the formation of a disk. Potential consequences are discussed in more detail below.

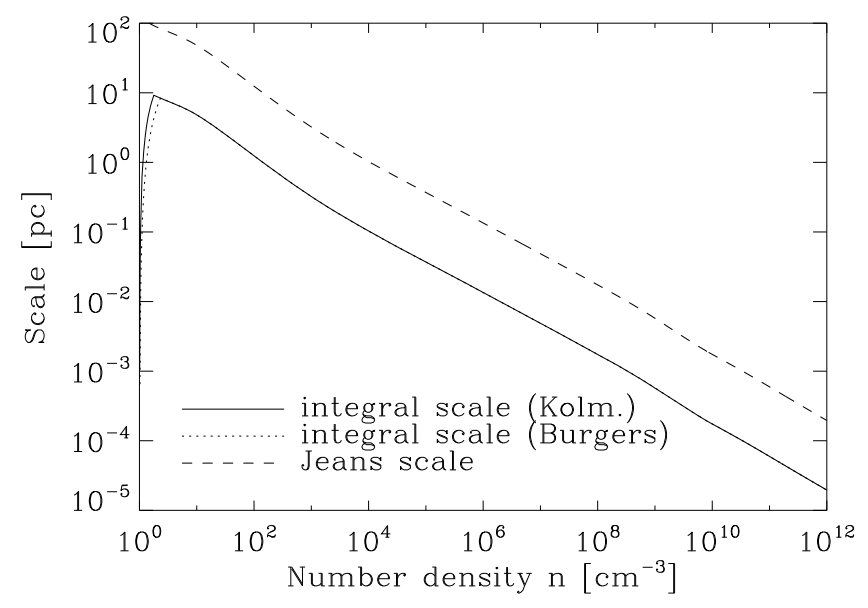

Fig. 8. The evolution of the integral scale of the magnetic field as a function of the average density reached in the central core at a given time during protostellar collapse in an atomic cooling halo with turbulent velocities of $\sim 20 \mathrm{~km} \mathrm{~s}^{-1}$ on the injection scale. We show cases corresponding to Kolmogorov- and Burgers-type turbulence and compare with the Jeans scale.

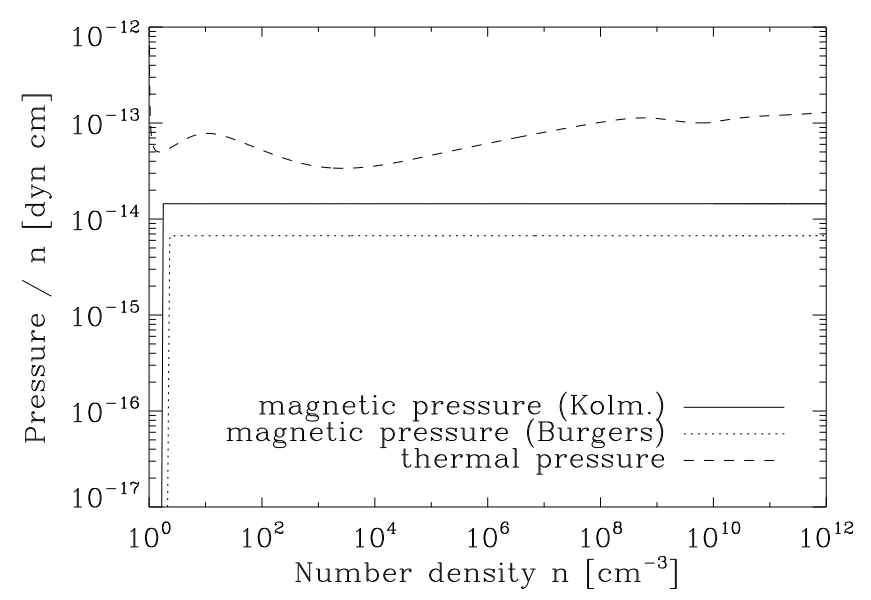

Fig. 9. Thermal and magnetic pressure as a function of the average density reached in the central core at a given time during protostellar collapse in aan atomic cooling halo with turbulent velocities of $\sim 20 \mathrm{~km} \mathrm{~s}^{-1}$ on the injection scale, for Kolmogorov- and Burgers-type turbulence.

\section{Discussion and conclusions}

We demonstrated in this paper that magnetic fields are generated rapidly by the small-scale dynamo both in minihalos which form the first stars, as well as in atomic cooling halos which may harbor the first galaxies. In this section, we summarize the main results and discuss the main consequences and open questions which cannot be resolved in this semi-analytic framework.

\subsection{Formation of the first stars}

The formation of the first stars was often examined by hydrodynamical simulations that neglected potential effects from magnetic fields (e.g. Abel et al. 2002; Bromm \& Larson 2004; Yoshida et al. 2006). Magnetic fields have however been considered to be important in the protostellar disk in presence of an efficient dynamo (Tan \& Blackman 2004; Silk \& Langer 2006). The impact of a uniformly imposed magnetic field $\left(B_{0}\right)$ in the primordial collapse has been studied by Machida et al. (2006, 2008) using direct numerical MHD simulations. In these simulations, a range of $B_{0}=10^{-6}-10^{-9} \mathrm{G}$ was used for different 
D. R. G. Schleicher et al.: Small-scale dynamo action during the formation of the first stars and galaxies.I.

values of the angular rotation velocity. A protostellar jet within a radius of about $0.02 \mathrm{AU}$ was found to be launched for an initial $B_{0} \geq 10^{-9} \mathrm{G}$ at $n=10^{3} \mathrm{~cm}^{-3}$. In this paper, we showed that the small-scale dynamo leads to a magnetic field strength much larger than the critical value of $10^{-9} \mathrm{G}\left(\mathrm{n} / 10^{3} \mathrm{~cm}^{-3}\right)$ derived by Machida et al. (2006) for the formation of jets and outflows. However, as these magnetic fields are more tangled than those of Machida et al. (2006), their results cannot be directly applied to ours, and additional numerical studies concerning tangled magnetic fields are required. The average Alfvén velocity $v_{\mathrm{A}}=B / \sqrt{\rho}$ is typically smaller than the sound speed, though it may dominate locally because of fluctuations in the magnetic field strength.

In addition, our study clarifies that the magnetic field strength in the protostellar disk is much higher than previously anticipated. The conditions for the MRI, as formulated by Tan \& Blackman (2004) and Silk \& Langer (2006), are thus fulfilled. This leads to the presence of turbulence in the protostellar disk, which may drive a large-scale $\alpha \omega$ dynamo in the presence of some kinetic helicity, making the field stronger and more coherent. But also the MRI itself may further amplify the magnetic field (Balbus \& Hawley 1991).

This has important consequences for the fragmentation behaviour of the disk. Detailed numerical studies of the collapse of magnetised molecular cloud cores in the context of present-day star formation (e.g. Hennebelle \& Teyssier 2008; Hennebelle \& Fromang 2008; Hennebelle \& Ciardi 2009; Mellon \& Li 2009) indicate that even modest field strengths can suppress binary formation and strongly favour the formation of single stars. Jets and magnetic tower flows are very effective in transporting away angular momentum and thus change structure and dynamics of the protostellar accretion disk. On the other hand, numerical simulations exploring the interaction of turbulence generated by the MRI with gravitational instabilities indicate the excitation of additional modes and an effective reduction of the accretion rate, as well as the broadening of spiral arms by the MRI turbulence (Fromang et al. 2004). Dedicated numerical studies exploring the combination of such effects in a primordial accretion disk will thus be required to understand the full impact on the stellar masses.

\subsection{Formation of the first galaxies}

The formation of the first galaxies is currently subject to much larger uncertainties than the formation of the first stars. This is because the initial conditions are not completely clear and the amount of metal enrichtment is not fully understood. The presence of supersonic turbulence has however been convincingly demonstrated by Greif et al. (2008) and Wise et al. (2008) with cosmological simulations encorporating hydrodynamics and primordial chemistry. Additional physics like supernova feedback may just enhance the amount of turbulence found there. The small-scale dynamo is found to be extremely efficient under these conditions and may magnetise the material during the collapse, with the magnetic pressure only half an order of magnitude below the thermal pressure on average. Locally, the magnetic field may even dominate in some places, as it is expected that highly inhomogeneous fields are generated from the smallscale dynamo. Indeed, as discussed by Subramanian (1999) and Brandenburg \& Subramanian (2005), the magnetic field is highly inhomogeneous, reaching equipartition in about $10 \%$ of the volume. The saturation field strength we adopted above results from a spatial average over the different local values. We thus expect fluctuations of the field strength by at least a factor of 10
(Wang \& Abel 2009; Dubois \& Teyssier 2009). Due to the increase of the integral scale, the magnetic field becomes more coherent in these systems, making a stronger case for the putative presence of jets and outflows.

As in the case of minihalos, the formation of a disk may lead to the presence of an $\alpha \omega$ dynamo that makes the magnetic field more coherent on disk scales. It may similarly play a role by making angular-momentum transport more efficient and thus reducing the amount of fragmentation and suppressing binary formation. As discussed above, the magnetic pressure may locally dominate over the thermal pressure. In this case, the magnetic Jeans mass sets the critical scale for fragmentation. For small-scale turbulent fields, it is defined in analogy to the thermal Jeans mass as

$$
M_{\mathrm{J}, B}=2 M_{\odot}\left(\frac{v_{\mathrm{A}}}{0.2 \mathrm{~km} \mathrm{~s}^{-1}}\right)^{3}\left(\frac{n}{10^{3} \mathrm{~cm}^{-3}}\right)^{-1 / 2} \propto \frac{B^{3}}{\rho^{2}} .
$$

Here, the Alfvén speed $v_{\mathrm{A}}=B / \sqrt{4 \pi \rho}$ replaces the sound speed $c_{\mathrm{s}}$, as magnetic pressure support propagates with the Alfvén speed. With

$v_{\mathrm{A}}=2.0 \mathrm{~km} \mathrm{~s}^{-1}\left(\frac{B}{10^{-5} \mathrm{G}}\right)\left(\frac{n}{10^{2} \mathrm{~cm}^{-3}}\right)^{-0.5}$,

the Alfvén speed in the saturation phase is thus larger than or comparable to the speed of sound.

The presence of such fields thus provides additional stability during the formation of intermediate-mass black holes, which are often considered to form in such systems (e.g. Eisenstein \& Loeb 1995; Koushiappas et al. 2004; Begelman et al. 2006; Spaans \& Silk 2006; Shang et al. 2009; Schleicher et al. 2010). Thus, even if fragmentation cannot be totally avoided in hydrodynamical simulations, the presence of magnetic fields may still give rise to larger seed masses. The detailed consequences however need to be assessed with numberical simulations. Additional open questions concern the further evolution of the magnetic field on larger scales and the build-up of galacticscale fields, as discussed by Arshakian et al. (2009). Their model for the magnetic-field evolution in galaxies yields a number of predictions which can be tested with future radio facilities such as the $\mathrm{SKA}^{1}$, which can thus constrain the formation mechanisms of the small- and large-scales magnetic fields in new born and young galaxies. In this respect, cosmological simulations that include an approximate treatment for the mean-field induction equation, as performed by Dubois \& Teyssier (2009), will be very important.

An additional issue that needs to be addressed is the role of magnetic helicity. Magnetic helicity is a conserved quantity and affects magnetic field generation and decay. In the presence of helical fields, the small-scale dynamo also creates correlations on larger scales (Subramanian 1998, 1999). The decay law for helical fields was derived by Hatori (1984). It is independent from the large scale part of the spectrum (i.e. scales above the integral scale) and is generally less efficient due to helicity conservation. During such decay, magnetic power is shifted from small to large scales via an inverse cascade, thus increasing the typical coherence length (Christensson et al. 2001). Our paper was conservative in the sense that we assumed magnetic fields with zero helicity, yielding a lower limit on the integral scale. In the presence of helicity, magnetic fields may be coherent on larger scales, making it more straightforward to drive large-scale jets and outflows. To assess this issue, the turbulent properties of

\footnotetext{
${ }^{1}$ http://www. skatelescope.org/
} 
the first galaxies need to be analyzed and understood in further detail.

\subsection{Further discussion}

Based on the estimates performed for this paper, it seems likely that the small-scale dynamo will be very efficient during the formation of the first stars and galaxies. The epoch of first star formation may thus also be the epoch where the first strong magnetic fields formed in the universe. This may be important for our understanding of primordial star formation. We speculate that this mechanism may not only apply to the very first galaxies, but that the formation of any gravitationally bound structures lead to a sufficient amount of accretion-driven turbulence to amplify magnetic fields. We plan to investigate this proposition further with numerical simulations.

Acknowledgements. We thank Axel Brandenburg, Daniele Galli, Marita Krause, Ralph Pudritz, Dmitry Sokoloff and Kandu Subramanian for stimulating discussions on the topic and the anonymous referee for helpful remarks on our manuscript. The research leading to these results has received funding from the European Community's Seventh Framework Programme (/FP7/2007-2013/) under grant agreement No 229517. R. Banerjee is funded by the Emmy-Noether grant (DFG) BA 3706/1-1. R.S.K. thanks the German Science Foundation (DFG) for support via the Emmy Noether grant KL 1358/1. D.R.G.S. and R.S.K. also acknowledge subsidies from the DFG SFB 439 Galaxies in the Early Universe. D.R.G.S., R.S.K., S.S. and T.G.A. thank for funding via the Priority Programme 1177 "Witnesses of Cosmic History: Formation and evolution of black holes, galaxies and their environment" of the German Science Foundation. In addition, R.S.K. thanks for subsidies from the German Bundesministerium für Bildung und Forschung via the ASTRONET project STAR FORMAT (grant 05A09VHA) and from the Landesstiftung Baden-Württemberg via their program International Collaboration II. R.S.K. also thanks the KIPAC at Stanford University and the Department of Astronomy and Astrophysics at the University of California at Santa Cruz for their warm hospitality during a sabbatical stay in spring 2010. KIPAC is sponsored in part by the US Department of Energy contract no. DEAC-02-76SF00515.

\section{References}

Abel, T., Anninos, P., Zhang, Y., \& Norman, M. L. 1997, New Astron., 2, 181 Abel, T., Bryan, G. L., \& Norman, M. L. 2002, Science, 295, 93

Arshakian, T. G., Beck, R., Krause, M., \& Sokoloff, D. 2009, A\&A, 494, 21

Balbus, S. A., \& Hawley, J. F. 1991, ApJ, 376, 214

Banerjee, R., \& Jedamzik, K. 2004, Phys. Rev. D, 70, 123003

Banerjee, R., \& Pudritz, R. E. 2007, ApJ, 660, 479

Banerjee, R., Pudritz, R. E., \& Anderson, D. W. 2006, MNRAS, 373, 1091

Banerjee, R., Vazquez-Semadeni, E., Hennebelle, P., \& Klessen, R. 2009, MNRAS, 398, 1082

Beck, R., Poezd, A. D., Shukurov, A., \& Sokoloff, D. D. 1994, A\&A, 289, 94

Begelman, M. C., Volonteri, M., \& Rees, M. J. 2006, MNRAS, 370, 289

Bertschinger, E. 1998, ARA\&A, 36, 599

Biskamp, D., \& Müller, W. 1999, Phys. Rev. Lett., 83, 2195

Brandenburg, A., Enqvist, K., \& Olesen, P. 1996, Phys. Rev. D, 54, 1291

Brandenburg, A., \& Subramanian, K. 2005, Phys. Rep., 417, 1

Bromm, V., \& Loeb, A. 2003, ApJ, 596, 34

Bromm, V., \& Larson, R. B. 2004, ARA\&A, 42, 79

Christensson, M., Hindmarsh, M., \& Brandenburg, A. 2001, PRE, 64, 056405

de Souza, R. S., \& Opher, R. 2010, Phys. Rev. D, 81, 067301

Dubois, Y., \& Teyssier, R. 2010, A\&A, 520, 17

Eisenstein, D. J., \& Loeb, A. 1995, ApJ, 443, 11

Federrath, C., Klessen, R. S., \& Schmidt, W. 2008, ApJ, 688, L79

Federrath, C., Roman-Duval, J., Klessen, R. S., Schmidt, W., \& Mac Low, M. 2010, A\&A, 512, A81
Fromang, S., Balbus, S. A., Terquem, C., \& De Villiers, J. 2004, ApJ, 616, 364 Galli, D., \& Palla, F. 1998, A\&A, 335, 403

Glover, S. 2005, Space Sci. Rev., 117, 445

Glover, S. C. O., \& Savin, D. W. 2009, MNRAS, 393, 911

Grasso, D., \& Rubinstein, H. R. 2001, Phys. Rep., 348, 163

Greif, T. H., Johnson, J. L., Klessen, R. S., \& Bromm, V. 2008, MNRAS, 387, 1021

Hatori, T. 1984, J. Phys. Soc. Japan, 53, 2539

Haugen, N. E. L., Brandenburg, A., \& Dobler, W. 2004a, Ap\&SS, 292, 53 Haugen, N. E. L., Brandenburg, A., \& Dobler, W. 2004b, PRE, 70, 016308 Haugen, N. E. L., Brandenburg, A., \& Mee, A. J. 2004c, MNRAS, 353, 947

Hennebelle, P., \& Fromang, S. 2008, A\&A, 477, 9

Hennebelle, P., \& Teyssier, R. 2008, A\&A, 477, 25

Hennebelle, P., \& Ciardi, A. 2009, A\&A, 506, L29

Kazantsev, A. P. 1968, Sov. Phys. JETP, 26, 1031

Klessen, R. S., \& Hennebelle, P. 2010, A\&A, 520, A17

Kolmogorov, A. 1941, Akademiia Nauk SSSR Doklady, 30, 301

Komatsu, E., Dunkley, J., Nolta, M. R., et al. 2009, ApJS, 180, 330

Koushiappas, S. M., Bullock, J. S., \& Dekel, A. 2004, MNRAS, 354, 292

Kritsuk, A. G., Norman, M. L., Padoan, P., \& Wagner, R. 2007, ApJ, 665, 416

Lazar, M., Schlickeiser, R., Wielebinski, R., \& Poedts, S. 2009, ApJ, 693, 1133

Machida, M. N., Omukai, K., Matsumoto, T., \& Inutsuka, S.-i. 2006, ApJ, 647, L1

Machida, M. N., Inutsuka, S.-i., \& Matsumoto, T. 2008, ApJ, 676, 1088

Maki, H., \& Susa, H. 2004, ApJ, 609, 467

Maki, H., \& Susa, H. 2007, PASJ, 59, 787

Medvedev, M. V., Silva, L. O., Fiore, M., Fonseca, R. A., \& Mori, W. B. 2004,

Journal of Korean Astronomical Society, 37, 533

Mee, A. J., \& Brandenburg, A. 2006, MNRAS, 370, 415

Mellon, R. R., \& Li, Z. 2009, ApJ, 698, 922

Omukai, K. 2001, ApJ, 546, 635

Pinto, C., \& Galli, D. 2008, A\&A, 484, 17

Pinto, C., Galli, D., \& Bacciotti, F. 2008, A\&A, 484, 1

Pudritz, R. E., \& Silk, J. 1989, ApJ, 342, 650

Ryu, D., Kang, H., Cho, J., \& Das, S. 2008, Science, 320, 909

Schekochihin, A. A., Cowley, S. C., Taylor, S. F., Maron, J. L., \& McWilliams, J. C. 2004, ApJ, 612, 276

Schleicher, D. R. G., Banerjee, R., \& Klessen, R. S. 2008a, Phys. Rev. D, 78, 083005

Schleicher, D. R. G., Galli, D., Palla, F., et al. 2008b, A\&A, 490, 521

Schleicher, D. R. G., Banerjee, R., \& Klessen, R. S. 2009a, ApJ, 692, 236

Schleicher, D. R. G., Galli, D., Glover, S. C. O., et al. 2009b, ApJ, 703, 1096

Schleicher, D. R. G., Galli, D., Glover, S. C. O., et al. 2009c, ApJ, 703, 1096

Schleicher, D. R. G., Spaans, M., \& Glover, S. C. O. 2010, ApJ, 712, L69

Schlickeiser, R., \& Shukla, P. K. 2003, ApJ, 599, L57

Schmidt, W., Federrath, C., Hupp, M., Kern, S., \& Niemeyer, J. C. 2009, A\&A, 494, 127

Seager, S., Sasselov, D. D., \& Scott, D. 1999, ApJ, 523, L1

Seager, S., Sasselov, D. D., \& Scott, D. 2000, ApJS, 128, 407

Sethi, S. K., \& Subramanian, K. 2005, MNRAS, 356, 778

Shang, C., Bryan, G. L., \& Haiman, Z. 2009, MNRAS, 1840

Silk, J., \& Langer, M. 2006, MNRAS, 371, 444

Smith, M. D., \& Mac Low, M. 1998, ApSS, 261, 191

Spaans, M., \& Silk, J. 2006, ApJ, 652, 902

Stancil, P. C., Lepp, S., \& Dalgarno, A. 1998, ApJ, 509, 1

Subramanian, K. 1998, MNRAS, 294, 718

Subramanian, K. 1999, Phys. Rev. Lett., 83, 2957

Subramanian, K., Shukurov, A., \& Haugen, N. E. L. 2006, MNRAS, 366, 1437

Sur, S., Schleicher, D. R. G., Banerjee, R., Federrath, C., \& Klessen, R. S. 2010, ApJ, 721, L134

Tan, J. C., \& Blackman, E. G. 2004, ApJ, 603, 401

Tashiro, H., \& Sugiyama, N. 2006, MNRAS, 372, 1060

Tashiro, H., Sugiyama, N., \& Banerjee, R. 2006, Phys. Rev. D, 73, 023002

Wang, P., \& Abel, T. 2009, ApJ, 696, 96

Wise, J. H., \& Abel, T. 2007, ApJ, 665, 899

Wise, J. H., Turk, M. J., \& Abel, T. 2008, ApJ, 682, 745

Xu, H., O'Shea, B. W., Collins, D. C., et al. 2008, ApJ, 688, L57

Yoshida, N., Omukai, K., Hernquist, L., \& Abel, T. 2006, ApJ, 652, 6

Yoshida, N., Omukai, K., \& Hernquist, L. 2008, Science, 321, 669 